\title{
Le cycle de réformes éducatives en Albanie : une transition vers les normes occidentales?
}

\section{Enkeleda Arapi et Frédéric Lasserre}

\section{OpenEdition}

12 Journals

Édition électronique

URL : https://journals.openedition.org/ries/5492

DOI : $10.4000 /$ ries.5492

ISSN : 2261-4265

Éditeur

France Education international

\section{Édition imprimée}

Date de publication : 1 septembre 2016

Pagination : 23-28

ISBN : 978-2-85420-611-1

ISSN : 1254-4590

\section{Référence électronique}

Enkeleda Arapi et Frédéric Lasserre, « Le cycle de réformes éducatives en Albanie : une transition vers les normes occidentales? », Revue internationale d'éducation de Sèvres [En ligne], 72 I septembre 2016, mis en ligne le 19 mars 2018, consulté le 03 juillet 2021. URL : http://journals.openedition.org/ries/ 5492 ; DOl : https://doi.org/10.4000/ries.5492

Ce document a été généré automatiquement le 3 juillet 2021.

(c) Tous droits réservés 


\title{
Le cycle de réformes éducatives en Albanie : une transition vers les normes occidentales?
}

\author{
Enkeleda Arapi et Frédéric Lasserre
}

1 Après la chute du régime communiste en 1991, l'Albanie a entrepris une profonde réforme de son système éducatif, à l'instar des réformes entreprises ailleurs en Europe centrale et orientale. Ces réformes étaient à la fois d'ordre politique, structurel et pédagogique, modifiant les programmes comme les approches pédagogiques, pour converger vers les normes occidentales.

2 L'Albanie, petit pays balkanique de l'Europe de l'Est, a une population de 2,9 millions d'habitants sur un territoire de $28748 \mathrm{~km}^{2}$. Environ $57,2 \%$ de la population est urbaine, avec un taux de chômage de $17,9 \%$ en 2013. Les minorités ethniques (vlach, grecque notamment) représentent environ $2 \%$ de la population et la langue officielle est l'albanais. Le pays a une histoire longue et unique qui intègre l'influence des cultures anciennes grec que et byzantine, romaine, et surtout ottomane, qui demeurent importantes dans la compréhension des systèmes de valeurs, de la culture, des traditions et de l'éducation. En 1944, pendant la Deuxième Guerre mondiale, le pays devient la République socialiste d'Albanie, suite à la prise de pouvoir par les communistes. Il fait partie du bloc dit de l'Est puis de meure l'un des pays les plus fermés d'Europe, après sa rupture avec Moscou, en1960. Le mouvement social et politique de la fin des années 1980, dans le bloc de l'Est, symbolisé par la chute du mur de Berlin en 1989, précipite la chute du régime communiste (1991) et influence considérablement la société albanaise. Le nouveau régime a entrepris des démarches politiques, sociales et économiques pour amorcer la transition du système communiste, au pouvoir pendant presque cinquante ans, vers une économie libérale.

3 Ainsi, depuis 1991, l'Albanie tente progressivement de mettre en place une société ouverte, une économie de marché et une forme de démocratie occidentale. Les changements traversent l'ensemble de la société, car tout ce qui est associé à l'ancien régime, dans les premières années qui suivent le changement politique, est 
systématiquement réformé, voire éliminé. Les bouleversements affectent donc également le secteur de l'éducation. Depuis deux décennies, les décideurs politiques ont tenté de transformer et de réorganiser le système éducatif, avec comme objectif affiché de le calquer le plus possible sur le modèle des pays occidentaux (West, 2013), à l'instar de ce qu'on observe dans les autres pays d'Europe centrale et orientale.

4 L'Albanie est candidate officielle à l'Union européenne (UE) depuis 2012. L'UE ne propose pas de directives éducatives communes, contrairement à d'autres secteurs (économie, politique, droit). Il s'avère cependant que, si l'UE ne réglemente pas le secteur éducatif - chaque État demeurant souverain dans ce domaine-, de grands principes de politique éducative, adressés à la plupart des pays en transition, sont tirés de recommandations sociales et économiques paneuropéennes, liées à la formation de la main-d'œuvre et à l'emploi. L'Albanie, comme la plupart des pays d'Europe centrale et orientale en transition, a été par conséquent fortement incitée à aligner ses politiques et pratiques éducatives sur celles qui sont mises en œuvre dans les pays membres de l'UE, tout comme l'ensemble des réformes administratives. De plus, dans la tourmente financière qui a suivi la chute du régime, l'Albanie a sollicité l'aide de l'UE et de la Banque mondiale, notamment dans le secteur éducatif : c'est aussi par le biais de ces programmes d'aide que l'UE et la Banque mondiale notamment, ont contribué à orienter les réformes éducatives.

5 Traditionnellement, les politiques liées au système éducatif en Albanie étaient fortement alimentées par la politique et l'idéologie du parti au pouvoir, au lieu de se fonder sur des éléments issus de la recherche scientifique, quasi inexistante, sous le régime communiste, dans le domaine des sciences de l'éducation (Whitehead, 2000) : les directives et l'organisation du système éducatif dépendaient autant d'orientations idéologiques que d'un souci d'efficacité et de formation de la main d'œuvre (West, 2013), mais aussi d'une transformation du système éducatif qui a permis d'éradiquer l'analphabétisme. De fait, très marqué par cet héritage idéologique, le système éducatif, aux yeux des nouveaux dirigeants soucieux de marquer une rupture avec l'ancien régime, se devait d'être vigoureusement réformé (Birzea, 1996 ; West, 2013). Cependant, dans de nombreux cas, les décideurs politiques ont mis en avant les réformes de l'éducation sans connaissance minimale de la recherche au sujet de leur propre système d'éducation ${ }^{1}$. La décision de réformer a souvent été prise sans une connaissance précise des forces et faiblesses de l'outil éducatif hérité de la période communiste : il fallait effacer toute approche administrative ou opérationnelle en vigueur avant 1991, sans nécessairement avoir une vision précise des objectifs éducatifs. Cette volonté de gommer un héritage rejeté relève tout autant de l'idéologie que celle de l'ancien régime, d'une certaine façon, en faisant prévaloir la finalité politique sur l'évaluation rationnelle des forces et faiblesses du secteur éducatif hérité de la période communiste (West, 2013).

6 Les réformes mises en place de 1991 à aujourd'hui ont été nombreuses et ont profondément modifié le système éducatif, le gouvernement albanais, cherchant à s'inspirer résolument d'un modèle occidental idéalisé, pour se démarquer radicalement du passé. Quelle a été la séquence des réformes et leurs effets sur le système éducatif albanais? Cet article tente de dépeindre le cheminement des réformes à travers leurs objectifs décidés par les gouvernements qui se sont succédé depuis 1991, dans un contexte de bouleversements politiques. 


\section{Les réformes du système éducatif albanais}

7 L'analyse des réformes promulguées depuis 1991 dans le domaine de l'éducation permet d'en retracer l'évolution. Cependant, on trouve peu de textes scientifiques sur les orientations des réformes éducatives post-1991 en Albanie.

La politique gouvernementale, depuis vingt ans, considère le champ de l'éducation comme une priorité nationale, à l'instar de la majorité des pays occidentaux. Depuis les années 1990, le système de l'éducation a subi des changements importants. Ceux-ci peuvent être regroupés en trois étapes.

\section{Purifier l'éducation des caractéristiques communistes}

Les années 1990 signent la fin d'un système communiste qui avait perduré pendant cinquante ans et l'implantation d'un système démocratique très fragile. Ce changement politique, social et culturel marque aussi des changements dans l'éducation nationale. Il conduit les nouvelles élites gouvernementales, même si elles sont parfois issues du Parti communiste, à remettre en cause les objectifs, le fonctionnement et les valeurs du système éducatif, ainsi que les programmes, la pédagogie, les valeurs, les objectifs et la culture de l'école. Pendant cette période, il s'agit principalement de «la "purification" du système de l'héritage et des effets de l'idéologie communiste » (MASH, 2014). Il faut adopter un curriculum libéré des manipulations politiques et des distorsions idéologiques, opérer une réduction relative de la charge de travail, trouver un meilleur équilibre entre les connaissances, les compétences et les attitudes, examiner les objectifs et le contenu des sciences sociales.

\section{Construire la base juridique du nouveau système éducatif (1995-2010)}

10 La deuxième étape visait à préparer la base juridique structurant le fonctionnement administratif du nouveau système. Au cours de cette période, le gouvernement approfondit la coopération avec des organisations étrangères, telles que la Banque mondiale, dont l'aide et l'expertise permettent la mise en œuvre de deux grands projets destinés à améliorer la qualité de l'enseignement (2000-2005; 2006-2010) et à préparer la stratégie nationale d'éducation. Les changements ont affecté la structure du système d'éducation désormais structuré ainsi :

- l'éducation préscolaire non obligatoire dure trois ans et est offerte aux enfants de 3-6 ans dans des garderies; pour les enfants de 5 ans qui n'ont pas suivi le programme préscolaire, des classes préparatoires (maternelles) sont intégrées dans les écoles primaires ;

- l'éducation de base dure neuf années (de 6 à 15 ans) et regroupe l'enseignement primaire et secondaire (cinq et quatre années respectivement) ;

- l'éducation secondaire supérieure concerne les élèves âgés de 15 à 18 ans: enseignement secondaire supérieur général, trois ans d'études ; enseignement secondaire spécialisé (trois à quatre ans), proposé dans des lycées généraux spécialisés dans les matières scientifiques et dont l'accès est sélectif, au mérite ; enseignement secondaire professionnel (quatre ans).

Outre la structure du cursus, la réforme a également touché les programmes et les structures de gestion. Malgré les efforts et les investissements, les évaluations des organismes nationaux et internationaux ont montré que la qualité du rendement des 
élèves demeure faible, tout comme l'efficacité du système, mesurée selon le taux de succès aux examens à la fin du secondaire ou selon le nombre d'élèves qui abandonnent l'école (MASH, 2014).

\section{Moderniser le système éducatif} démocratique, en tenant compte des exigences des principes de base de l'égalité des chances en matière de qualité de l'éducation et de respect des différences individuelles : il ne s'agit plus de purger les traces du passé communiste ni de refonder les bases administratives du secteur éducatif, mais d'étoffer le contenu des programmes et d'en moderniser les approches pédagogiques. Un objectif important de cette période consiste à réformer la gouvernance de l'éducation, en particulier par le biais de la décentralisation, très lacunaire selon les normes des pays occidentaux (West, 2013). Un autre objectif de cette phase est d'améliorer la qualité des processus d'enseignement par le biais de la modernisation du programme, du développement professionnel des enseignants, de l'amélioration des manuels scolaires et des méthodes d'évaluation des élèves, notamment.

centres d'intérêt de l'apprenant, radicalement différent du système traditionnel, qui obligeait l'élève à s'adapter à la mécanique du cours magistral centré sur le maître. C'est un système éducatif où les élèves et les enseignants travaillent ensemble, où la connaissance du domaine de l'apprentissage et les compétences sont construits par les élèves, qui produisent des solutions à des problèmes complexes pour l'individu et pour la société ; un système qui valorise l'ouverture internationale via l'enseignement de deux langues étrangères et des technologies de l'information et de la communication pour pré les apprenants à faire face aux défis de l'environnement mondial ; un système qui valorise l'enseignement continu ${ }^{2}$.

14 Au-delà de ces vœux pieux, l'objectif de la réforme est d'établir les meilleures conditions possibles pour les élèves, indépendamment des capacités et de l'origine sociale (principe de l'égalité des chances), pour, en théorie, préparer et former des citoyens actifs capables de participer de façon créative et critique à la vie économique, sociale et culturelle du pays et de s'adapter à l'évolution d'une société démocratique. Si le principe de l'égalité des chances demeure ainsi inchangé depuis l'époque communiste, les objectifs diffèrent en ce que la réforme vise à former des citoyens actifs et adaptés à un marché de l'emploi régi par des principes libéraux.

De fait, les objectifs des dernières réformes albanaises rejoignent largement les finalités des réformes mises en œuvre dans les pays occidentaux. Ces dernières sont essentiellement "une adaptation des systèmes éducatifs aux besoins éducatifs d'une société donnée ; une amélioration des conditions d'apprentissage, une adéquation des formations aux exigences de l'emploi et un épanouissement des personnes diplômées ", avec des réformes fondées sur une logique de compétence, une centration sur l'apprenant et une importance accrue accordée à la formation des enseignants (Jonnaert, Lafortune, Ettayebi, 2007). 


\section{Les objectifs des dernières réformes}

\section{Une logique de compétence et une centration sur l'apprenant}

16 Dans le curriculum de l'école albanaise, les compétences sont encore en construction mais sont inspirées des programmes occidentaux : France, Suisse, Angleterre, Finlande, Québec ${ }^{3}$. Elles sont articulées autour de sept axes: communication; raisonnement; apprendre à apprendre; mode de vie, entrepreneuriat et environnement ; compétence d'ordre personnel et social; savoir-vivre ensemble et citoyenneté; technologies de l'information. Ces éléments sont communs à nombre de programmes scolaires occidentaux.

17 Janaqi souligne que le curriculum traditionnel d'avant les réformes était bien davantage centré sur les enseignants et sur l'acquisition des savoirs académiques; la structure du curriculum était rigide, très centralisée et peu flexible, mettant l'accent sur la mémorisation et la reproduction exacte de connaissances préfabriquées. Or le nouveau curriculum repose sur la valorisation des processus d'apprentissage et de la méthodologie transdisciplinaire (logique de compétence), tout en montrant une flexibilité dans la structure et la répartition du temps, ouvert à la communauté et à la formation continue.

\section{L'importance accordée à la formation des maîtres}

La profession d'enseignant est contrôlée et les exigences sont élevées (MASH, 2014) Pour avoir le droit de l'exercer, chaque candidat doit avoir: terminé des études de deuxième cycle en pédagogie de la discipline de spécialité de l'enseignant; suivi un stage de pratique professionnelle d'environ un an dans les établissements d'enseignement complémentaire ; passé l'examen d'État pour les enseignants dans son profil.

La préparation des enseignants, malgré les changements apportés dans la formation, qui comporte des connaissances et des compétences théoriques appropriées, a cependant besoin d'être améliorée (MASH, 2014). Or, leur formation initiale ou continue accorde peu de place à l'acquisition d'expérience de la pratique quotidienne de l'enseignement en classe, souvent insuffisante; dans certains cas, elle présente même des lacunes car le renforcement de la capacité des enseignants à faire face aux facteurs externes à l'école, comme la participation des parents et de la communauté, n'est tout simplement pas pris en considération. Il est cependant important que la formation initiale et continue des maîtres tienne davantage compte du besoin de compétences nécessaires pour un enseignement plus adapté à un environnement social qui a connu d'importantes mutations (Whitehead, 2000). Le même auteur, en s'intéressant à la structuration des stages dans la formation initiale des maitres, dans le cadre des réformes en éducation, souligne que, suite à la promulgation des réformes, les universités ont restructuré leurs programmes de formation des maîtres, en augmentant le temps consacré aux stages professionnels. Il mentionne cependant que l'application de la réforme a été lente et influencée également par la crise politique albanaise de 1997, ainsi que par la guerre au Kosovo (1999). En outre, ces événements ont rendu difficile l'évaluation des progrès de la réforme pendant cette période et la communication entre chercheurs albanais et universités européennes. 
En somme, la nature et les objectifs visés par les réformes en éducation entreprises par le gouvernement albanais, ces dernières années, se rapprochent beaucoup de la typologie des réformes en éducation en Europe centrale et orientale (Whitehead, 2000) :

- réformes visant à corriger les caractéristiques les plus apparentes de l'éducation conçue à l'époque communiste ;

- réformes systémiques, impliquant un changement de paradigme : la décentralisation et la définition du rôle de l'État pour permettre l'émergence d'une démocratie pluraliste, la poursuite des principes de l'économie de marché dans les organisations éducatives et la mise en œuvre du contrôle de la qualité par une évaluation et la responsabilité publique ;

- réformes de modernisation visant à adapter les programmes, les manuels, l'équipement et les méthodes d'enseignement;

- réformes structurelles qui concernent des changements dans la durée des études et entre les différents niveaux et types d'enseignement.

Le processus de réforme en Albanie "a été avant tout un processus bottomup » (Whitehead, 2000). Il semble que le ministère de l'éducation a été impliqué dans ce processus, mais ne l'a pas dirigé, les réformes marquant une rupture avec l'ancien système de contrôle centralisé.

Il importe d'évaluer les forces et les faiblesses de ces réformes, en ce qui concerne la pratique des enseignants.

Tableau 1. Statistiques économiques : budget et éducation

\begin{tabular}{|l|c|c|c|c|c|c|}
\hline & $\begin{array}{c}\text { PIB } \\
\text { (millions USD, } \\
\text { milliards leks) }\end{array}$ & $\begin{array}{c}\text { Budget } \\
\text { de létat } \\
\text { (millions USD, } \\
\text { milliards leks) }\end{array}$ & $\begin{array}{c}\text { Budget } \\
\text { de lÉtat } \\
\text { en éducation } \\
\text { (millions USD, } \\
\text { milliards leks) }\end{array}$ & $\begin{array}{c}\text { Part du budget } \\
\text { en éducation } \\
\text { dans le budget } \\
\text { dePÉtat }\end{array}$ & $\begin{array}{c}\text { Budget en } \\
\text { éducation, } \\
\% \text { du PIB }\end{array}$ & $\begin{array}{c}\text { Dépenses } \\
\text { des ménages } \\
\text { en éducation, } \\
\% \text { du PIB }\end{array}$ \\
\hline 2009 & $\begin{array}{c}12313 \\
(1,148,1)\end{array}$ & $\begin{array}{c}4074 \\
(379,9)\end{array}$ & $\begin{array}{c}421 \\
(39,3)\end{array}$ & $10,3 \%$ & $3,42 \%$ & $0,8 \%$ \\
\hline 2013 & $\begin{array}{c}12851 \\
(1358)\end{array}$ & $\begin{array}{c}3876 \\
(409,6)\end{array}$ & $\begin{array}{c}402 \\
(42,5)\end{array}$ & $10,4 \%$ & $3,13 \%$ & $0,9 \%$ \\
\hline $\begin{array}{l}2015 \\
\text { (plan) }\end{array}$ & $\begin{array}{c}11432 \\
(1492,1)\end{array}$ & $\begin{array}{c}3639 \\
(475)\end{array}$ & $\begin{array}{c}342 \\
(44,6)\end{array}$ & $9,5 \%$ & $3,1 \%$ & $0,9 \%$ \\
\hline
\end{tabular}

Source : Ministria e Arsimit dhe Sportit (2016), Dokumenti I Strategjisë Së Zhvillimit Të Arsimit Parauniversitar [Ministère de l'Éducation et des Sports, Document de la stratégie du développement de l'éducation pré-universitaire] 2014-2020, Tirana.

Tableau 2. Effectifs dans l'enseignement public, 2014

\begin{tabular}{|l|c|c|c|c|}
\hline & Préscolaire & Primaire & $\begin{array}{c}\text { Secondaire } \\
1^{\text {er }} \text { cycle }\end{array}$ & $\begin{array}{c}\text { Secondaire } \\
2^{e} \text { cycle }\end{array}$ \\
\hline Elèves & 77154 & 177177 & 115609 & 165567 \\
\hline Enscignants & 4151 & 9389 & 7138 & 13516 \\
\hline Institutions & 1751 & 1317 & & 381 \\
\hline Directeurs & 202 & 1110 & & 381 \\
\hline Ratio enseignants/élèves & $1: 19$ & $1: 15$ & & $1: 16$ \\
\hline
\end{tabular}

Source : Ministria e Arsimit dhe Sportit, Dokumenti I Strategjisë Së Zhvillimit Të Arsimit Parauniversitar (2016) [Ministère de l'Éducation et des Sports, Document de la stratégie du développement de l'éducation pré-universitaire] 2014-2020, Tirana. 


\section{BIBLIOGRAPHIE}

BIRZEA C. (1996) : « Education in a World in Transition: Between Post-communism and Postmodernism », Prospects, $n^{\circ} 26$ (4), p. 673-681.

JONNAERT P., LAFORTUNE L., ETTAYEBI M. (2007) : « Un regard sur les réformes en éducation », in L. Lafortune, M. Ettayebi, P. Jonnaert, Observer les réformes en éducation, Québec : Presses de l'Université du Québec, p. 1-14.

MASH [Ministère de l'éducation et des sciences] (2014) : Grupi I Punës Për Reformimin E Arsimit Parauniversitar - Reforma E Sistemit Arsimor Parauniversitar. Raporti Paraprak. [Groupe de travail pour la réforme en éducation préuniversitaire - Réforme du système éducatif préuniversitaire, rapport préliminaire], Tirana.

WEST J. (2013) : Vocational Education and Training in Eastern Europe: Transition and Influence, Centre for Learning and Life Chances in Knowledge Economies and Societies [en ligne] [http:// www.llakes.org/wp-content/uploads/2013/04/41.-West-2.pdf]

WHITEHEAD J.(2000) : « Teacher education reforms in Albania », European journal of teacher education, $\mathrm{n}^{\circ} 1$, p. 85-94.

\section{NOTES}

1. Sahlberg P., Boce E. (2010) : «Are teachers teaching for a knowledge society?», Teachers and Teaching: theory and practice, 16 (1), p. 31-48.

2. Janaqi G. (2014) : «Le cadre curriculaire en enseignement de la République de l'Albanie» (Korniza Kurrikulare E Arsimit Parauniversitar Të Republikës Së Shqipërisë), Revista pedagogike, 2014, p. 29-105.

3. Janaqi 2014, op. cit.

\section{INDEX}

Mots-clés : curriculum, réforme de l'enseignement, système scolaire, formation des enseignants Palabras claves : curriculum, reforma de la educación, sistema escolar, formación de docentes Index géographique : Albanie

Keywords : curriculum, educational reform, school system, teacher education

\section{AUTEURS}

\section{ENKELEDA ARAP}

Enkeleda Arapi est Doctorante au département d'études sur l'enseignement et l'apprentissage à l'Université de Laval (Québec). Ses travaux portent sur la collaboration des acteurs éducatifs en 
enseignement primaire. Diplômée de l'Université de Laval en psychologie et psychopédagogie et en enseignement primaire à l'Université Alexandre Xhuvani (Albanie), elle enseigne dans les programmes de l'éducation au préscolaire et de l'enseignement primaire à l'Université de Laval. Courriel : Enkeleda.arapi@fse.ulaval.ca

\section{FRÉDÉRIC LASSERRE}

Frédéric Lasserre est titulaire d'un MBA de l'Université de York, d'un DEA en géopolitique de l'Université Paris 8 et d'un doctorat de géographie de l'Université de Saint-Étienne. Il a travaillé à l'Observatoire européen de géopolitique (OEG, Lyon) sur les transformations politiques et économiques de l'Europe centrale et orientale après 1991, comme conseiller en affaires internationales au ministère québécois de l'industrie et du commerce, puis au sein d'Investissement Québec. Il est professeur depuis 2001 au département de géographie de l'Université de Laval (Québec), et chercheur à l'Institut québécois des Hautes études internationales (HEI). Il est également directeur du Conseil québécois d'études géopolitiques (CQEG) aux HEI. Courriel : Frederic.lasserre@ggr.ulaval.ca 